\title{
Dynamical instability of shear-free collapsing star in extended teleparallel gravity
}

\author{
Abdul Jawad ${ }^{\mathrm{a}}$, Shamaila Rani ${ }^{\mathrm{b}}$ \\ Department of Mathematics, COMSATS Institute of Information Technology, Lahore 54000, Pakistan
}

Received: 10 September 2015 / Accepted: 18 October 2015 / Published online: 24 November 2015

(C) The Author(s) 2015. This article is published with open access at Springerlink.com

\begin{abstract}
We study the spherically symmetric collapsing star in terms of dynamical instability. We take the framework of extended teleparallel gravity with a non-diagonal tetrad, a power-law form of the model presenting torsion and a matter distribution as a non-dissipative anisotropic fluid. The vanishing shear scalar condition is adopted to gain insight in a collapsing star. We apply a first order linear perturbation scheme to the metric, the matter, and $f(T)$ functions. The dynamical equations are formulated under this perturbation scheme to develop collapsing equation for finding dynamical instability limits in two regimes, such as the Newtonian and the post-Newtonian regime. We obtain a constraint-free solution of a perturbed time dependent part with the help of a vanishing shear scalar. The adiabatic index exhibits the instability ranges through the second dynamical equation which depend on physical quantities such as the density, the pressure components, the perturbed parts of the symmetry of the star, etc. We also develop some constraints on the positivity of these quantities and obtain instability ranges to satisfy the dynamical instability condition.
\end{abstract}

\section{Introduction}

The gravitational collapse of self-gravitating objects has become a widely discussed subject in general relativity (GR) as well as in modified theories of gravity. This contains the evolutionary development and constancy of these objects during the collapse process and is importantly at the center of studies of structure formation. This process occurs when a stable matter becomes unbalanced and ultimately undergoes a collapse, which results in different structures like stars, stellar groups, and planets. In this way, self-gravitating objects go across various dynamical states, which may be analyzed

\footnotetext{
a e-mail: jawadab181@yahoo.com; abduljawad@ciitlahore.edu.pk

b e-mail: shamailatoor.math@yahoo.com;

drshamailarani@ciitlahore.edu.pk
}

through dynamical equations. The dynamical instability was first investigated by Chandrasekhar [1] with the help of the adiabatic index $\Gamma$ of a spherical star with isotropic pressure. This index depicts the consequences of various structural quantities of a fluid on the instability ranges. For instability ranges in the Newtonian and post-Newtonian regimes, Herrera et al. [2] explored a dissipative, non-adiabatic spherically symmetric collapsing star.

The adiabatic index develops the instability ranges in GR as well as in modified theories of gravity, which induces these ranges to depend on dark source terms in addition to the usual terms. Under different conditions for cylindrically and spherically symmetric collapsing matters in $f(R)$ gravity, the instability ranges have been explored through the adiabatic index [3-9]. Taking the expansion-free condition, Skripkin [10] developed a model for a non-dissipative spherically symmetric fluid distribution with isotropy and constant energy density and remarked that a Minkowskian cavity is observed at the center of fluid. Under this condition, the instability for spherically and cylindrically symmetric anisotropic fluids in Newtonian and post-Newtonian regimes is explored in GR [11] as well as $f(R)$ gravity [14,15]. In Brans-Dicke gravity, Sharif and Manzoor [16] explored the instability ranges of a spherically symmetric collapsing star.

The physical aspects such as isotropy, radiation, anisotropy, shear, dissipation, and expansion are the main sources driving the gravitational evolution. Among these factors, the shear leads to the formation of naked singularities. That is, it contributes to the formation of an apparent horizon, which results in a black hole of the evolving cloud. Thus, the shear tensor points to the direction of a good motivation to study structure formation and its consequences on the dynamical instability range of a self-gravitating body.

In the context of extended teleparallel gravity (ETG) (or $f(T)$ gravity) which is the generalization of teleparallel gravity, the gravitational collapse is discussed with and without expansion scalar by Sharif and Rani [17,18]. They found that the physical properties have a vast impact on the dynamical 
instability in studying the self-gravitating objects with expansion. Without expansion, they obtain the instability ranges for the Newtonian $(\mathrm{N})$ and post-Newtonian $(\mathrm{pN})$ regimes. In this paper, we assume a shear-free condition instead an expansion-free one and explore the instability ranges of a collapsing star in ETG.

The scheme of the paper is given as follows. In Sect. 2, we give the basics of ETG and provide the construction of the field equations in two ways, simple and covariant form. Section 3 contains the basic equations for the static spherically symmetry. Also, junction conditions are given for the dynamical instability of a spherically symmetric collapsing star in the context of ETG gravity. In the next section, we represent a perturbation scheme and an ETG model and apply it to all matter, metric, and $f(T)$ functions. In Sect. 5, we formulate a dynamical collapsing equation and find the instability ranges in the $\mathrm{N}$ and $\mathrm{pN}$ regimes. The last section summarizes the results and elaborates a comparison.

\section{Extended teleparallel gravity}

In this section, we provide the basics, such as the tetrad field and the Weitzenböck connection of ETG. We give the field equations in simple form as well as their covariant construction.

\subsection{Tetrad field}

The geometry of ETG is unambiguously described through an orthonormal set having three spacelike and one timelike fields, called the tetrad field. The trivial tetrad field has the form $e_{a}=\delta_{a}^{\mu} \partial_{\mu}, e^{b}=\delta_{\mu}^{b} \mathrm{~d} x^{\mu}$, where $\delta_{\mu}^{a}$ is the Kronecker delta. This is the simplest field and less important due to the zero torsion. The non-trivial tetrad field allows for a non-zero torsion and grants the construction of teleparallel as well as ETG theory. It is given by

$h_{a}=h_{a}{ }^{\mu} \partial_{\mu}, \quad h^{b}=h^{b}{ }_{\nu} \mathrm{d} x^{\nu}$,

satisfying the following properties:

$h^{a}{ }_{\mu} h_{b}^{\mu}=\delta_{b}^{a}, \quad h^{a}{ }_{\mu} h_{a}{ }^{v}=\delta_{\mu}^{v}$.

The metric tensor is demonstrated as a by-product of this field, which is as follows:

$g_{\mu \nu}=\eta_{a b} h_{\mu}^{a} h_{\nu}^{b}$

\subsection{The Weitzenböck connection}

The basic phenomenon of teleparallel gravity and ETG is the parallel transport of the tetrad field in Weitzenböck space- time, which is carried out by the significant component Weitzenböck connection.

By applying a covariant derivative w.r.t. spacetime of tetrad field, we get

$\Delta_{\nu} h_{\mu}^{a}=\partial_{\nu} h_{\mu}^{a}-\widetilde{\Gamma}_{\mu \nu}^{\alpha} h_{\alpha}^{a} \equiv 0$,

where $\widetilde{\Gamma}_{\mu \nu}^{\alpha}=h_{a}{ }^{\alpha} \partial_{\nu} h^{a}{ }_{\mu}$ is the Weitzenböck connection. We obtain the torsion tensor by the antisymmetric part of this connection as follows:

$T_{\mu \nu}^{\alpha}=\widetilde{\Gamma}_{\nu \mu}^{\alpha}-\widetilde{\Gamma}_{\mu \nu}^{\alpha}=h_{a}^{\alpha}\left(\partial_{\nu} h_{\mu}^{a}-\partial_{\mu} h^{a}{ }_{\nu}\right)$,

which is antisymmetric in its lower indices, i.e., $T_{\mu \nu}^{\alpha}=$ $-T^{\alpha}{ }_{\nu \mu}$. This absolute parallelism rapidly removed the curvature of the Weitzenböck connection identically. The following relation is also satisfied by the Weitzenböck connection:

$\widetilde{\Gamma}_{\mu \nu}^{\alpha}=\Gamma_{\mu \nu}^{\alpha}+K_{\mu \nu}^{\alpha}$,

here $\Gamma_{\mu \nu}^{\alpha}, K^{\alpha}{ }_{\mu \nu}$ appear as the usual Levi-Civita connection (torsionless) and the contorsion tensor, respectively, which can be defined as follows:

$$
\begin{aligned}
\Gamma_{\mu \nu}^{\alpha} & =\frac{1}{2} g^{\alpha \rho}\left[g_{\rho \nu, \mu}+g_{\rho \mu, \nu}-g_{\mu \nu, \rho}\right], \\
K_{\mu \nu}^{\alpha} & =\frac{1}{2}\left[T_{\mu}^{\alpha}{ }_{\nu}+T_{\nu}{ }^{\alpha}{ }_{\mu}-T_{\mu \nu}^{\alpha}\right] .
\end{aligned}
$$

\subsection{Field equations}

To generalize the action of teleparallel gravity, we just substitute a general function of torsion scalar by itself as follows [19-25]:

$\mathcal{S}=\frac{1}{2 \kappa^{2}} \int h\left(f(T)+\mathcal{L}_{m}\right) \mathrm{d}^{4} x$

where $h=\operatorname{det}\left(h_{\lambda}^{a}\right), \mathcal{L}_{m}$ is the matter Lagrangian and $f$ is a function of the torsion scalar. The torsion scalar is defined as

$T=S_{\alpha}^{\mu \nu} T_{\mu \nu}^{\alpha}$,

where

$S_{\alpha}^{\mu \nu}=\frac{1}{2}\left[-\frac{1}{2}\left(T_{\alpha}^{\mu \nu}-T_{\alpha}^{\nu \mu}-T_{\alpha}^{\mu \nu}\right)+\delta_{\alpha}^{\mu} T^{\theta v}{ }_{\theta}-\delta_{\alpha}^{v} T^{\theta \mu}\right]$

is called the superpotential tensor. Applying the variation of the action (9) w.r.t. a tetrad field, we will get the field equations as follows:

$\left[\frac{1}{h} \partial_{\mu}\left(h h_{a}{ }^{\alpha} S_{\alpha}{ }^{\mu v}\right)+h_{a}{ }^{\alpha} T_{\mu \alpha}^{\lambda} S_{\lambda}{ }^{v \mu}\right] f_{T}+h_{a}{ }^{\alpha} S_{\alpha}{ }^{\mu v} \partial_{\mu} T f_{T T}$ 


$$
+\frac{1}{4} h_{a}{ }^{\nu} f=\frac{1}{2} \kappa^{2} h_{a}{ }^{\alpha} \mathcal{T}_{\alpha}^{v}
$$

where $f_{T}$ is the first order derivative and $f_{T T}$ represents second order derivative of $f$ with respect to $T$.

The field equations (12) turn out to be extremely different from Einstein's equations on account of the tetrad components and the partial derivatives. Since tetrads are not completely eradicated, this causes difficulty to compare teleparallel gravity (and ETG) with GR. To obtain an equivalent description of the field equations (12) to the other modified theories, we will apply the covariant formalism [26-29]. We replace all partial derivatives in Eqs. (5), (8), and (11) by covariant derivatives using the condition on metric tensor, $\nabla_{\sigma} g_{\mu \nu}=0$, i.e., the compatibility of the metric tensor, we get

$$
\begin{aligned}
T_{\mu \nu}^{\alpha}= & h_{a}^{\alpha}\left(\partial_{\mu} h_{\nu}^{a}-\Gamma_{\mu \nu}^{\sigma} h_{\sigma}^{a}-\partial_{\nu} h_{\mu}^{a}+\Gamma_{\mu \nu}^{\sigma} h_{\sigma}^{a}\right) \\
= & h_{a}^{\alpha}\left(\nabla_{\mu} h_{\nu}^{a}-\nabla_{\nu} h_{\mu}^{a}\right), \\
K_{\mu \nu}^{\alpha}= & h_{a}^{\alpha} \nabla_{\nu} h_{\mu}^{a}, \quad S_{\alpha}{ }^{\mu}=\eta^{a b} h_{a}^{\mu} \nabla_{\alpha} h_{b}^{v}+\delta_{\alpha}^{\nu} \eta^{a b} h_{a}^{\sigma} \nabla_{\sigma} h_{b}^{\mu} \\
& -\delta_{\alpha}^{\mu} \eta^{a b} h_{a}^{\sigma} \nabla_{\sigma} h_{b}^{v},
\end{aligned}
$$

where we have applied the following relations:

$S^{\mu(\nu \alpha)}=T^{\mu(\nu \alpha)}=K^{(\mu \nu) \alpha}=0$.

In this case, the Weitzenböck connection becomes zero, while the Riemann tensor turns out to be

$$
\begin{aligned}
R_{\mu \rho \nu}^{\alpha} & =\partial_{\rho} \Gamma_{\mu \nu}^{\alpha}-\partial_{\nu} \Gamma_{\mu \rho}^{\alpha}+\Gamma^{\alpha}{ }_{\sigma \rho} \Gamma_{\mu \nu}^{\sigma}-\Gamma^{\alpha}{ }_{\sigma \nu} \Gamma^{\sigma}{ }_{\mu \rho} \\
& =\nabla_{\nu} K_{\mu \rho}^{\alpha}-\nabla_{\rho} K_{\mu \nu}^{\alpha}+K^{\alpha}{ }_{\sigma \nu} K_{\mu \rho}^{\sigma}-K^{\alpha}{ }_{\sigma \rho} K_{\mu \nu}^{\sigma} .
\end{aligned}
$$

The corresponding Ricci tensor becomes

$R_{\mu \nu}=\nabla_{\nu} K_{\mu \alpha}^{\alpha}-\nabla_{\alpha} K_{\mu \nu}^{\alpha}+K_{\rho \nu}^{\alpha} K_{\mu \alpha}^{\rho}-K_{\rho \alpha}^{\alpha} K_{\mu \nu}^{\rho}$.

Using Eq. (13) along with $S_{\alpha \nu}^{\nu}=-2 T^{\nu}{ }_{\alpha \nu}=2 K^{\nu}{ }_{\alpha \nu}$, we have

$$
\begin{aligned}
R_{\mu \nu} & =-\nabla^{\alpha} S_{\nu \alpha \mu}-g_{\mu \nu} \nabla^{\alpha} T^{\rho}{ }_{\alpha \rho}-S^{\alpha \rho}{ }_{\mu} K_{\rho \alpha \nu}, \\
R & =-T-2 \nabla^{\alpha} T^{\nu}{ }_{\alpha \nu} .
\end{aligned}
$$

The covariant derivative of torsion tensor in the last equation shows the only difference between Ricci and torsion scalars.

After some calculations, we will get

$G_{\mu \nu}-\frac{1}{2} g_{\mu \nu} T=-\nabla^{\alpha} S_{\nu \alpha \mu}-S^{\sigma \alpha}{ }_{\mu} K_{\alpha \sigma \nu}$,

where $G_{\mu \nu}=R_{\mu \nu}-\frac{1}{2} g_{\mu \nu} R$ appears as the Einstein tensor. By using this in (12), we attain the required field equations in $f(T)$ gravity,

$f_{T} G_{\mu \nu}+\frac{1}{2} g_{\mu \nu}\left(f-T f_{T}\right)+D_{\mu \nu} f_{T T}=\kappa^{2} \mathcal{T}_{\mu \nu}$,

here $D_{\mu \nu}=S_{\nu \mu}{ }^{\alpha} \nabla_{\alpha} T$. It can be observed that (17) has an equivalent structure such as $f(R)$ gravity and reduces to GR for $f(T)=T$. Here, the trace of the above equation is
$D f_{T T}-(R+2 T) f_{T}+2 f=\kappa^{2} \mathcal{T}$,

with $D=D^{v}{ }_{v}$ and $\mathcal{T}=\mathcal{T}^{v}{ }_{v}$. The $f(T)$ field equations can also be defined as

$G_{\mu \nu}=\frac{\kappa^{2}}{f_{T}}\left(\mathcal{T}_{\mu \nu}^{m}+\mathcal{T}_{\mu \nu}^{T}\right)$

Here $\mathcal{T}_{\mu \nu}^{m}$ represents the matter fluid and the torsion contribution is

$\mathcal{T}_{\mu \nu}^{T}=\frac{1}{\kappa^{2}}\left[-D_{\mu \nu} f_{T T}-\frac{1}{4} g_{\mu \nu}\left(\mathcal{T}-D f_{T T}+R f_{T}\right)\right]$.

\section{Basic equations}

The general spherically symmetric metric in the interior region is

$\mathrm{d} s_{-}^{2}=X^{2} \mathrm{~d} t^{2}-Y^{2} \mathrm{~d} r^{2}-R^{2}\left(\mathrm{~d} \theta^{2}+\sin ^{2} \theta \mathrm{d} \phi^{2}\right)$,

where $X, Y$, and $R$ are functions of $t$ and $r$. The line element for the exterior spacetime (the Schwarzschild metric) is [14, $15]$

$\mathrm{d} s_{+}^{2}=\left(1-\frac{2 M}{r}\right) \mathrm{d} v^{2}+2 \mathrm{~d} r \mathrm{~d} v-r^{2}\left(\mathrm{~d} \theta^{2}+\sin ^{2} \theta \mathrm{d} \phi^{2}\right)$,

where $v$ is the retarded time and $M$ represents the total mass of the bounded surface. Also, the anisotropic energymomentum tensor can be defined as follows:

$\mathcal{T}_{\nu}^{\mu}=\left(\rho+p_{\perp}\right) u^{\mu} u_{\nu}-p_{\perp} \delta_{\nu}^{\mu}+\left(p_{r}-p_{\perp}\right) v^{\mu} v_{\nu}$,

in the interior region, while $\rho=\rho(t, r), p_{r}=p_{r}(t, r), p_{\perp}=$ $p_{\perp}(t, r)$. The four velocity $u_{\mu}=\frac{1}{X} \delta_{\mu}^{0}$ and unit four vector directed toward the radial component $v_{\mu}=\frac{1}{Y} \delta_{\mu}^{1}$ satisfy the relations $u_{\mu} u^{\mu}=1, v_{\mu} v^{\mu}=-1, u_{\mu} v^{\mu}=0$. We take the non-diagonal tetrad for the interior spacetime as

$h^{i}{ }_{\mu}$

$$
=\left(\begin{array}{cccc}
X & 0 & 0 & 0 \\
0 & Y \sin \theta \cos \phi & R \cos \theta \cos \phi & -R \sin \theta \sin \phi \\
0 & Y \sin \theta \sin \phi & R \cos \theta \sin \phi & R \sin \theta \cos \phi \\
0 & Y \cos \theta & -R \sin \theta & 0
\end{array}\right) .
$$

The description of a gravitational collapsing star takes on the kinematics of the dynamical equations of spherically symmetric models. This needs acceleration, expansion, rotation, and distortion or shear. Due to the shear-free condition, there have been published many interesting results such as that this condition depicts the physical aspects of compact 
bodies in the relativistic astrophysics phenomena. The shear scalar and tensor are defined by

$\sigma^{2}=\frac{1}{2} \sigma^{\alpha \beta} \sigma_{\alpha \beta}$,

where

$$
\begin{aligned}
& \sigma_{\alpha \beta}=V_{(\alpha ; \beta)}+a_{(a} V_{\beta)}-\frac{1}{3} \Theta\left(g_{\alpha \beta}+V_{\alpha} V_{\beta}\right), \quad \Theta=V_{; \alpha}^{\alpha}, \\
& a=V_{(\alpha ; \beta)} V^{\beta} .
\end{aligned}
$$

For the interior spacetime, this tensor yields the following scalar:

$\sigma=\frac{1}{X}\left(\frac{\dot{Y}}{Y}-\frac{\dot{R}}{R}\right)$

Using the tetrad along with Eq. (21) in (19), the field equations are

$$
\begin{aligned}
& \left(\frac{2 \dot{Y}}{Y}+\frac{\dot{R}}{R}\right) \frac{\dot{R}}{R}-\left(\frac{X}{Y}\right)^{2}\left[\frac{2 R^{\prime \prime}}{R}+\left(\frac{R^{\prime}}{R}\right)^{2}-\frac{2 Y^{\prime} R^{\prime}}{Y R}-\left(\frac{Y}{R}\right)^{2}\right] \\
& =\frac{\kappa^{2}}{f_{T}}\left[\rho X^{2}+\frac{X^{2}}{\kappa^{2}}\left\{\frac{T f_{T}-f}{2}-\frac{1}{Y^{2}}\left(\frac{R^{\prime}}{R}-\frac{Y}{R}\right) f_{T}^{\prime}\right\}\right], \\
& 2\left(\frac{\dot{R}^{\prime}}{R}-\frac{\dot{R} X^{\prime}}{R X}-\frac{\dot{Y} R^{\prime}}{Y R}\right)=\frac{\dot{R} f_{T}^{\prime}}{R f_{T}}, \\
& 2\left(\frac{\dot{R}{ }^{\prime}}{R}-\frac{\dot{R} X^{\prime}}{Y R}-\frac{\dot{Y} R^{\prime}}{Y R}\right)=\frac{\dot{T}}{T^{\prime}}\left(\frac{R^{\prime}}{R}-\frac{Y}{R}\right) \frac{f_{T}^{\prime}}{f_{T}}, \\
& \quad-\left(\frac{Y}{X}\right)^{2}\left[\frac{2 \ddot{R}}{R}-\left(\frac{2 \dot{X}}{X}-\frac{\dot{R}}{R}\right) \frac{\dot{R}}{R}\right]+\left(\frac{2 X^{\prime}}{X}+\frac{R^{\prime}}{R}\right) \frac{R^{\prime}}{R}-\left(\frac{Y}{R}\right)^{2} \\
& =\frac{\kappa^{2}}{f_{T}}\left[p_{r} Y^{2}-\frac{Y^{2}}{\kappa^{2}}\left(\frac{T f_{T}-f}{2}+\frac{\dot{R} \dot{T} f_{T}^{\prime}}{X^{2} R T^{\prime}}\right)\right], \\
& \quad-\left(\frac{R}{X}\right)^{2}\left[\frac{\ddot{Y}}{Y}+\frac{\ddot{R}}{R}-\frac{\dot{X}}{X}\left(\frac{\dot{Y}}{Y}+\frac{\dot{R}}{R}\right)+\frac{\dot{Y} \dot{R}}{Y R}\right]+\left(\frac{R}{Y}\right)^{2}\left[\frac{X^{\prime \prime}}{X}+\frac{R^{\prime \prime}}{R}\right. \\
& \left.\quad-\frac{X^{\prime} Y^{\prime}}{X Y}+\left(\frac{X^{\prime}}{X}-\frac{Y^{\prime}}{Y}\right) \frac{R^{\prime}}{R}\right]=\frac{\kappa^{2}}{f_{T}}\left[p_{\perp} R^{2}-\frac{R^{2}}{\kappa^{2}}\left\{\frac{T f_{T}-f}{2}+\frac{1}{2}\right.\right. \\
& \left.\left.\quad \times\left(\frac{1}{X^{2}}\left(\frac{\dot{Y}}{Y}+\frac{\dot{R}}{R}\right) \frac{\dot{T}}{T^{\prime}}-\frac{1}{Y^{2}}\left(\frac{X^{\prime}}{X}+\frac{R^{\prime}}{R}-\frac{Y}{R}\right)\right) f_{T}^{\prime}\right\}\right] .
\end{aligned}
$$

The torsion scalar takes the form

$$
\begin{aligned}
T= & 2\left[\frac{2}{X Y R}\left(\frac{X^{\prime} R^{\prime}}{Y}-\frac{\dot{Y} \dot{R}}{X}\right)-\frac{1}{X^{2}}\left(\frac{\dot{R}}{R}\right)^{2}+\frac{1}{Y^{2}}\left(\frac{R^{\prime}}{R}\right)^{2}+\frac{1}{R^{2}}\right. \\
& \left.-\frac{2}{Y R}\left(\frac{X^{\prime}}{X}+\frac{R^{\prime}}{R}\right)\right] .
\end{aligned}
$$

Also, from Eqs. (29) and (30), we obtain a relationship as follows:

$\frac{\dot{R}}{R}=\frac{\dot{T}}{T^{\prime}}\left(\frac{R^{\prime}}{R}+\frac{Y}{R}\right)$.

To analyze the properties of collapsing star, the non-trivial contracted identities yield the dynamical equations, which are very useful. These are given by

$$
\left(\stackrel{m}{\mathcal{T}^{\mu \nu}}+\stackrel{T}{\mathcal{T}^{\mu \nu}}\right)_{; \nu} u_{\mu}=0, \quad\left(\stackrel{m}{\mathcal{T}^{\mu \nu}}+\stackrel{T}{\mathcal{T}^{\mu \nu}}\right)_{; \nu} v_{\mu}=0 .
$$

Using these equations, the dynamical equations become

$$
\begin{aligned}
& {\left[\frac{\dot{\rho}}{X}+\left(\rho+p_{r}\right) \frac{\dot{Y}}{X Y}+2\left(\rho+p_{\perp}\right) \frac{\dot{R}}{X R}\right]} \\
& \quad+\frac{X}{\kappa^{2}}\left[\left(\frac{T f_{T}-f}{2 X^{2}}-\frac{\dot{R} T^{\prime} f_{T}^{\prime}}{X^{2} Y^{2} R \dot{T}}\right)_{, 0}\right. \\
& \quad+\frac{\dot{X}}{X^{3}}\left(T f_{T}-f\right)+\left(\frac{\dot{R} f_{T}^{\prime}}{X^{2} Y^{2} R}\right)_{, 1}-\frac{1}{X^{2} R}\left\{\frac{2 \dot{R}}{X Y^{2}}\left(\frac{\dot{X} T^{\prime}}{\dot{T}}-2 X^{\prime}\right)+\frac{\dot{R}}{X^{2}}\right. \\
& \left.\left.\quad \times\left(\frac{2 \dot{Y}}{Y}+\frac{\dot{R}}{R}\right) \frac{\dot{T}}{T^{\prime}}+\frac{\dot{R}}{Y^{2}}\left(\left(\frac{\dot{R}}{R}+\frac{\dot{Y}}{Y}\right) \frac{T^{\prime}}{\dot{T}}-\frac{Y^{\prime}}{Y}-\frac{2 R^{\prime}}{R}\right)\right\} f_{T}^{\prime}\right]=0,
\end{aligned}
$$

$\left[\frac{p_{r}^{\prime}}{Y}+\left(\rho+p_{r}\right) \frac{X^{\prime}}{X Y}+2\left(p_{r}-p_{\perp}\right) \frac{R^{\prime}}{Y R}\right]+\frac{Y}{\kappa^{2}}\left[\left(\frac{\dot{R} f_{T}^{\prime}}{X^{2} Y^{2} R}\right)_{, 0}-\frac{1}{Y^{2}}\right.$

$$
\begin{aligned}
& \times\left(\frac{Y^{\prime}}{Y}+\frac{R^{\prime}}{R}\right)\left(T f_{T}-f\right)+\left(\frac{f-T f_{T}}{2 Y^{2}}-\frac{\dot{R} \dot{T} f_{T}^{\prime}}{X^{2} Y^{2} R T^{\prime}}\right)_{, 1}+\frac{1}{X Y^{2} R} \\
& \times\left\{-\frac{X^{\prime} \dot{R} T^{\prime}}{Y^{2} \dot{T}}+\frac{\dot{R}}{X}\left(\frac{3 \dot{Y}}{Y}+\frac{\dot{X}}{X}\right)+2 \frac{\dot{R}^{2}}{X R}\left(1-\frac{1}{Y^{2}}\right)-\frac{\dot{R}}{X Y}\right. \\
& \left.\left.\times\left(Y^{\prime}+\frac{Y^{\prime}}{Y^{2}}+\frac{X^{\prime}}{X Y}\right) \frac{\dot{T}}{T^{\prime}}\right\} f_{T}^{\prime}\right]=0 .
\end{aligned}
$$

\subsection{Junction conditions}

In order to join smoothly the interior and exterior spacetimes over a hypersurface $\Sigma^{(e)}$, we apply junction conditions. The collapse problems are dealt with by Darmois junction conditions in an appropriate manner. These conditions require the continuity of intrinsic and extrinsic curvatures over the hypersurface, i.e., $\left(\mathrm{d} s^{2}\right)_{\Sigma}=\left(\mathrm{d} s_{-}^{2}\right)_{\Sigma}=\left(\mathrm{d} s_{+}^{2}\right)_{\Sigma}$ and $\mathcal{K}_{a b}=\mathcal{K}_{a b}^{-}=\mathcal{K}_{a b}^{+}$, respectively. The Misner-Sharp mass function is given by

$m(t, r)=\frac{R}{2}\left(1+g^{\mu \nu} R_{, \mu} R_{, \nu}\right)$,

where a spherical object of radius $R$ contributes a total energy, and this contributes to our study of the Darmois junction conditions. For Eq. (21), it takes the form

$m(t, r)=\frac{R}{2}\left(1+\frac{\dot{R}^{2}}{X^{2}}-\frac{R^{\prime 2}}{Y^{2}}\right)$.

In order to match exterior region with interior, it is required that $r=r_{\Sigma^{(e)}}=$ constant on the boundary surface $\Sigma^{(e)}$ $[14,15,30]$, which results in

$M \stackrel{\Sigma^{(e)}}{=} m(t, r)$

$2\left(\frac{\dot{R}^{\prime}}{R}-\frac{\dot{R} X^{\prime}}{R X}-\frac{\dot{Y} R^{\prime}}{Y R}\right) \stackrel{\Sigma^{(e)}}{=}-\frac{Y}{X}\left[\frac{2 \ddot{R}}{R}-\left(\frac{2 \dot{X}}{X}-\frac{\dot{R}}{R}\right) \frac{\dot{R}}{R}\right]$ 


$$
+\frac{X}{Y}\left[\left(\frac{2 X^{\prime}}{X}+\frac{R^{\prime}}{R}\right) \frac{R^{\prime}}{R}-\left(\frac{Y}{R}\right)^{2}\right] .
$$

Substituting the field equations (29) and (31) in the above equation, we get

$-p_{r} \stackrel{\Sigma^{(e)}}{=} \frac{\mathcal{T}_{11}^{T}}{Y^{2}}-\frac{\mathcal{T}_{01}^{T}}{X Y}=\frac{f\left(T_{c}\right)}{2}$,

where $f\left(T_{c}\right)$ represents a constant value for constant torsion scalar $T_{c}$.

\section{Linear perturbation strategy and power-law $f(T)$ model}

In $f(R)$ gravity, the gravitational collapse is widely discussed, taking a power-law form of model, which is simply generalized GR. We take in particular the $f(T)$ model in power-law form analogy to the $f(R)$ model $[14,15]$, like $f(R)=R+\gamma R^{2}$, to analyze the evolution of the collapsing star. The power-law $f(T)$ model has contributed as a most viable model due to its simple form and we may directly compare our results with GR. We assume the ETG model as follows:

$f(T)=T+\delta T^{2}$,

where $\delta$ is an arbitrary constant. For this model, we obtain an accelerated expansion universe in the phantom phase, we have the possibility of realistic wormhole solutions and instability conditions for a collapsing star. We assume the linear perturbation strategy to construct the dynamical equations in order to explore instability ranges for the underlying scenario. For this purpose, we assume the system initially to be in static equilibrium. That is, metric and matter parts are at zeroth order perturbation only radially dependent, which also become time dependent for the first order perturbations. These perturbations are described as follows [3-18,30-33]:

$$
\begin{aligned}
X(t, r) & =X_{0}(r)+\epsilon \Pi(t) x(r), \\
Y(t, r) & =Y_{0}(r)+\epsilon \Pi(t) y(r), \\
R(t, r) & =R_{0}(r)+\epsilon \Pi(t) c(r), \\
\rho(t, r) & =\rho_{0}(r)+\epsilon \hat{\rho}(t, r), \\
p_{r}(t, r) & =p_{r 0}(r)+\epsilon \hat{p}_{r}(t, r), \\
p_{\perp}(t, r) & =p_{\perp 0}(r)+\epsilon \hat{p}_{\perp}(t, r), \\
m(t, r) & =m_{0}(r)+\epsilon \hat{m}(t, r), \\
T(t, r) & =T_{0}(r)+\epsilon \Pi(t) e(r),
\end{aligned}
$$

where the quantities with zero subscript denote the static parts of corresponding functions and $0<\epsilon \ll 1$. The perturbed vanishing shear scalar and the $f(T)$ model takes the form

$$
\begin{aligned}
f(T) & =T_{0}\left(1+\delta T_{0}\right)+\epsilon \Pi e\left(1+2 \delta T_{0}\right), \\
f_{T}(T) & =1+2 \delta T_{0}+2 \epsilon \delta \Pi e, \\
\frac{y}{Y_{0}} & =\frac{c}{R_{0}} .
\end{aligned}
$$

Taking into account shear-free condition $(\sigma=0)$, Eq. (26) yields $\frac{\dot{Y}}{Y}=\frac{\dot{R}}{R}$. The solution of this equation turns out to be $Y=\alpha R$ where $\alpha$ is an arbitrary function of $r$ taken as 1 without loss of generality; and using the freedom to rescale the radial coordinate, we take $R_{0}=r$, which is also the Schwarzschild coordinate. The condition under which an initially shear-free flow remains shear-free all along the evolution has been studied by Herrera et al. [34]. One of the consequences of such a study is that the pressure anisotropy may affect the propagation in time, of the shear-free condition. The shear-free condition is unstable, in particular, against the presence of pressure anisotropy. An expansion scalar and a scalar function ensured the departures from the shear-free condition for the geodesic case. These scalars are defined in purely physical variables such as in terms of the Weyl tensor, anisotropy of pressure, and the shear viscosity. It is remarked that one can consider such a case that pressure anisotropy and density inhomogeneity are present in such a way that the scalar function appearing in an orthogonal splitting of the Riemann tensor vanishes, implying a non-homogeneous anisotropic stable shear-free flow. Since we are dealing with a fluid evolving under the shear-free condition, so we shall make use of this condition while evaluating the components of the field equations and also in the conservation equations.

Now we evaluate the zeroth order as well as first order configurations. The zeroth order perturbation of the field equations (28)-(31) is given by

$$
\begin{aligned}
& \frac{1}{1+2 \delta T_{0}}\left[\kappa^{2} \rho_{0}+\delta\left(\frac{T_{0}^{2}}{2}-\frac{2 c T_{0}^{\prime 2}}{e r Y_{0}^{2}}\right)\right] \\
& =\frac{1}{\left(Y_{0} r\right)^{2}}\left(2 r \frac{Y_{0}^{\prime}}{Y_{0}}+Y_{0}^{2}-1\right), \\
& \frac{1}{1+2 \delta T_{0}}\left[\kappa^{2} p_{r 0}-\delta \frac{T_{0}^{2}}{2}\right]=\frac{1}{\left(Y_{0} r\right)^{2}}\left(2 r \frac{X_{0}^{\prime}}{X_{0}}-Y_{0}^{2}+1\right), \\
& \frac{1}{1+2 \delta T_{0}}\left[\kappa^{2} p_{\perp 0}-\delta \frac{T_{0}^{2}}{2}+\frac{\delta T_{0}^{\prime}}{Y_{0}^{2}}\left(\frac{X_{0}^{\prime}}{X_{0}}+\frac{c T_{0}^{\prime}}{r e}\right)\right] \\
& =\frac{1}{Y_{0}^{2}}\left[\frac{X_{0}^{\prime \prime}}{X_{0}}-\frac{X_{0}^{\prime}}{X_{0}} \frac{Y_{0}^{\prime}}{Y_{0}}+\frac{1}{r}\left(\frac{X_{0}^{\prime}}{X_{0}}-\frac{Y_{0}^{\prime}}{Y_{0}}\right)\right] .
\end{aligned}
$$

The first dynamical equation (36) fulfills the zeroth order perturbation identically, while the second dynamical equation (36) becomes

$$
\frac{2}{r}\left(p_{r 0}-p_{\perp 0}\right)+p_{r 0}^{\prime}+\frac{X_{0}^{\prime}}{X_{0}}\left(\rho_{0}+p_{r 0}\right)
$$




$$
-\frac{\delta}{\kappa^{2}}\left[T_{0} T_{0}^{\prime}+\frac{T_{0}^{2}}{r}+\frac{2 c X_{0}^{\prime} T_{0}^{\prime 2}}{e X_{0} Y_{0}^{2} r}\right]=0
$$

In a static background, the matching condition, mass function, and torsion scalar contribute for a static equilibrium thus:

$$
\begin{aligned}
& p_{r 0} \stackrel{\Delta^{(e)}}{=} \frac{\delta T_{0}^{2}}{2 \kappa^{2}}, \quad m_{0}(r)=\frac{r}{2}\left(1-\frac{1}{Y_{0}^{2}}\right), \\
& T_{0}=\frac{2}{r}\left[\frac{1}{r}\left(1-\frac{3}{Y_{0}}\right)-\frac{1}{Y_{0}}\left(\frac{2 X_{0}^{\prime}}{X_{0}}+\frac{1}{r}\right)\left(1-\frac{1}{Y_{0}}\right)\right] .
\end{aligned}
$$

Applying the perturbed quantities to the field equations, we obtain

$$
\begin{aligned}
& -\frac{2 \Pi}{Y_{0}^{2}}\left[\left(\frac{c}{r}\right)^{\prime \prime}-\frac{1}{r}\left(\frac{y}{Y_{0}}\right)^{\prime}-\left(\frac{Y_{0}^{\prime}}{Y_{0}}-\frac{3}{r}\right)\left(\frac{c}{r}\right)^{\prime}\right] \\
& =\frac{2 \Pi c}{Y_{0}^{2} r^{3}}\left(2 r \frac{Y_{0}^{\prime}}{Y_{0}}+Y_{0}^{2}-1\right)+\frac{\kappa^{2} \hat{\rho}}{1+2 \delta T_{0}}+\frac{\Pi \delta}{\left(1+2 \delta T_{0}\right)} \\
& \times\left[T_{0} e-\frac{2 \kappa^{2} \rho_{0} y}{1+2 \delta T_{0}}-\frac{\delta T_{0}^{2} y}{1+2 \delta T_{0}}-\frac{c T_{0}^{\prime}}{e Y_{0}^{2} r}\left(2 e^{\prime}-\frac{4 \delta T_{0}^{\prime} e}{1+2 \delta T_{0}}-\frac{4 c T_{0}^{\prime}}{r}\right)\right], \\
& \left(\frac{c}{r}\right)^{\prime}-\left(\frac{X_{0}^{\prime}}{X_{0}}-\frac{1}{r}\right) \frac{c}{r}-\frac{c}{r^{2}}=\frac{\delta T_{0}^{\prime} c}{r\left(1+2 \delta T_{0}\right)}, \\
& \left(\frac{c}{r}\right)^{\prime}-\left(\frac{X_{0}^{\prime}}{X_{0}}-\frac{1}{r}\right) \frac{c}{r}-\frac{c}{r^{2}}=\frac{e\left(1-Y_{0}\right) \delta}{r\left(1+2 \delta T_{0}\right)}, \\
& -\frac{2 \ddot{\Pi} Y_{0}^{2} c}{r X_{0}^{2}}+\frac{2 \Pi}{r}\left[\left(\frac{x}{X_{0}}\right)^{\prime}+\left(r \frac{X_{0}^{\prime}}{X_{0}}+1\right)\left(\frac{c}{r}\right)^{\prime}\right] \\
& =\frac{\kappa^{2} Y_{0}^{2} \hat{p}_{r}}{1+2 \delta T_{0}}+\frac{2 \kappa^{2} \Pi Y_{0}^{2}}{1+2 \delta T_{0}}\left(\frac{c}{r}-\frac{\delta e}{1+2 \delta T_{0}}\right) p_{r} \\
& -\frac{\delta \Pi T_{0} Y_{0}^{2}}{1+2 \delta T_{0}}\left(e+\frac{c T_{0}}{r}-\frac{\delta Y_{0} T_{0} e}{1+2 \delta T_{0}}\right) \text {, } \\
& -\frac{2 c \ddot{\Pi}}{r X_{0}^{2}}+\frac{\Pi}{Y_{0}^{2}}\left[\left(\frac{x}{X_{0}}\right)^{\prime \prime}+\left(\frac{c}{r}\right)^{\prime \prime}+\left(\frac{2 X_{0}^{\prime}}{X_{0}}-\frac{Y_{0}^{\prime}}{Y_{0}}+\frac{1}{r}\right)\left(\frac{x}{X_{0}}\right)^{\prime}\right. \\
& \left.-\left(\frac{Y_{0}^{\prime}}{Y_{0}}-\frac{1}{r}\right)\left(\frac{c}{r}\right)^{\prime}\right]=\frac{\kappa^{2} \hat{p}_{\perp}}{1+2 \delta T_{0}} \\
& -\frac{2 \Pi e \delta \kappa^{2}}{\left(1+2 \delta T_{0}\right)^{2}} p_{\perp 0}+\frac{2 \Pi c}{r Y_{0}^{2}}\left[\frac{X_{0}^{\prime \prime}}{X_{0}}-\frac{X_{0}^{\prime}}{X_{0}} \frac{Y_{0}^{\prime}}{Y_{0}}+\frac{1}{r}\left(\frac{X_{0}^{\prime}}{X_{0}}-\frac{Y_{0}^{\prime}}{Y_{0}}\right)\right] \\
& +\frac{\delta \Pi}{1+2 \delta T_{0}}\left[\frac{e \delta T_{0}^{2}}{1+2 \delta T_{0}}-e T_{0}+\frac{X_{0}^{\prime} e^{\prime}}{X_{0} Y_{0}^{2}}-\frac{2 c T_{0}^{\prime} X_{0}^{\prime}}{r X_{0} Y_{0}^{2}}-\frac{2 e T_{0}^{\prime} X_{0}^{\prime} \delta}{X_{0} Y_{0}^{2}\left(1+2 \delta T_{0}\right)}\right. \\
& \left.-\frac{a X_{0}^{\prime} T_{0}^{\prime}}{X_{0}^{2} Y_{0}^{2}}+\frac{x^{\prime} T_{0}^{\prime}}{X_{0} Y_{0}^{2}}+\frac{e^{\prime} c^{2} T_{0}^{\prime}}{e r^{2} Y_{0}^{2}}-\frac{2 c T_{0}^{\prime}}{r^{2} Y_{0}^{2}}-\frac{2 c \delta T_{0}^{\prime 2}}{r Y_{0}^{2}\left(1+2 \delta T_{0}\right)}\right] \text {. }
\end{aligned}
$$

Applying Eqs. (42)-(47) to the Bianchi identities, it follows that

$\dot{\hat{\rho}}+\left[\left(3 \rho_{0}+p_{r 0}+2 p_{\perp 0}\right) \frac{c}{r}+J_{0}\right] \dot{\Pi} 0$,

where

$$
J_{0}=\frac{1}{\kappa^{2}}\left[\delta T_{0} e-\frac{4 c T_{0}^{\prime} \delta}{e r Y_{0}^{2}}\left(e^{\prime}-T_{0}^{\prime}\left(\frac{x}{X_{0}}+\frac{2 c}{r}\right)\right)+\frac{2 \delta}{X_{0}^{2}}\left(\frac{c T_{0}^{\prime}}{X_{0}^{2} Y_{0}^{2} r}\right)_{, 1}\right.
$$

$$
\left.-\frac{4 c \delta T_{0}^{\prime}}{X_{0} Y_{0}^{2} r}\left(\frac{x T_{0}^{\prime}}{e}-2 X_{0}^{\prime}\right)+\frac{2 c \delta T_{0}^{\prime}}{r Y_{0}^{2}}\left\{\frac{2}{r}+\frac{Y_{0}^{\prime}}{Y_{0}}-\frac{2 c T_{o}^{\prime}}{e r}\right\}\right] .
$$

Integrating Eq. (64) with respect to time, we have

$\hat{\rho}=-\left[\left(3 \rho_{0}+p_{r 0}+2 p_{\perp 0}\right) \frac{c}{r}+J_{0}\right] \Pi$.

The perturbed second Bianchi identity is given by

$$
\begin{gathered}
p_{r 0}^{\prime}-\left(\rho_{0}+p_{r 0}\right)\left(\frac{x}{X_{0}}\right)^{\prime} \frac{r}{c}+\left(\rho_{0}+p_{r 0}\right) \frac{X_{0}^{\prime}}{X_{0}}-\left(\hat{\rho}+\hat{p}_{r}\right) \frac{X_{0}^{\prime} r}{X_{0} \Pi r}+J_{1} \\
-2\left(p_{r 0}-p_{\perp 0}\right)\left(\frac{r}{c}\left(\frac{c}{r}\right)^{\prime}-\frac{1}{r}\right)-\left(\hat{p}_{r}-\hat{p}_{\perp}\right) \frac{2}{\Pi c}-\frac{c}{\Pi r} \hat{p}_{r}^{\prime}=0,
\end{gathered}
$$

where

$$
\begin{aligned}
J_{1}= & \frac{r \delta}{c \kappa^{2}}\left[Y_{0}^{2}\left(\frac{T_{0} e}{Y_{0}^{2}}-\frac{T_{0}^{2} c}{r Y_{0}^{2}}\right)_{, 1}-\frac{2 T_{0}^{\prime} c}{r X_{0}^{2}} \frac{\ddot{\Pi}}{\Pi}+\frac{2 T_{0} Y_{0}^{\prime} e}{Y_{0}}-\frac{3 c Y_{0}^{\prime} T_{0}^{2}}{r Y_{0}}\right. \\
& +\left(\frac{c Y_{0}}{r}\right)^{\prime} \frac{T_{0}^{2}}{Y_{0}}-\frac{2 c T_{0}^{2}}{r^{2}}+\frac{2 T_{0} e}{r}+T_{0}^{2}\left(\frac{c}{r}\right)^{\prime}+\frac{2 c x^{\prime} T_{0}^{\prime 2}}{e r X_{0} Y_{0}^{2}} \\
& \left.+\frac{2 c X_{0}^{\prime} T_{0}^{\prime}}{e r X_{0} Y_{0}^{2}}\left(1-\frac{m_{0}}{r}\right)^{\prime}\left(1-\frac{m_{0}}{r}\right)\left(2 e^{\prime}-\frac{x T_{0}^{\prime}}{X_{0}}-\frac{4 c T_{0}^{\prime}}{r}\right)\right] .
\end{aligned}
$$

The junction condition, the torsion scalar, and the mass function are

$$
\begin{aligned}
\hat{p_{r}} \stackrel{\Sigma^{(e)}}{=} & \frac{\delta}{\kappa^{2}}\left(\Pi T_{0} e-\frac{2 T_{0}^{\prime} \dot{\Pi} c}{r X_{0} Y_{0}}\right), \\
e= & \frac{4}{r Y_{0}^{2}}\left[\frac{1}{X_{0} Y_{0}}\left\{x^{\prime}+X_{0}^{\prime} c^{\prime}-\frac{X_{0}^{\prime}}{Y_{0}}\left(y+\frac{x}{X_{0}}+\frac{y}{Y_{0}}+\frac{c}{r}\right)\right\}\right. \\
& \left.-\frac{c Y_{0}}{r^{2}}+\frac{x X_{0}^{\prime}}{X_{0}^{2}}-\frac{x^{\prime}}{X_{0}}-\left(\frac{c}{r}\right)^{\prime}+\left(\frac{y}{Y_{0}}+\frac{c}{r}\right)\left(\frac{X_{0}^{\prime}}{X_{0}}+\frac{2}{r}\right)\right],
\end{aligned}
$$

$\bar{m}=-\frac{\Pi}{Y_{0}^{2}}\left[r\left(c^{\prime}-\frac{y}{Y_{0}}\right)+\frac{c}{2}\left(1-Y_{0}^{2}\right)\right]$.

Substituting zeroth order and first order matching conditions in Eq. (62), we have

$\ddot{\Pi} \stackrel{\Sigma^{(e)}}{=} 0$

showing the general solution of this equation is

$\Pi(t)=h_{1} t+h_{2}$,

where $h_{1}$ and $h_{2}$ are arbitrary constants. It is remarked that we do not need to impose any extra condition on this solution due to a vanishing shear scalar condition for collapse to occur. In [3-9, 14-18], we need to apply some constraint for the static solution in order to discuss instability analysis. 


\section{Collapse equation and dynamical instability}

In this section, we construct the collapse equation in order to work for dynamical instability in different regimes with shear-free condition. The Harison-Wheeler equation of state is used in this regard which is given by $[35,36]$

$$
\hat{p}_{i r}=\hat{\rho} \frac{p_{i 0}}{\rho_{0}+p_{i 0}} \Gamma .
$$

We use this index in order to examine instability ranges in the context of ETG with $\sigma=0$. The adiabatic index $\Gamma$ finds the rigidity of the fluid and evaluates the change of pressure to the corresponding density. Substituting the value of $\hat{\rho}$, it follows that

$$
\begin{aligned}
& \hat{p}_{r}=-\Pi\left[\frac{2 c}{r} \frac{\rho_{0}+p_{\perp 0}}{\rho_{0}+p_{r 0}} p_{r 0}+\frac{c}{r} p_{r 0}+\frac{p_{r 0}}{\rho_{0}+p_{r 0}} J_{0}\right] \Gamma, \\
& \hat{p}_{\perp}=-\Pi\left[\frac{c}{r} \frac{\rho_{0}+p_{r 0}}{\rho_{0}+p_{\perp 0}} p_{\perp 0}+\frac{2 c}{r} p_{\perp 0}+\frac{p_{\perp 0}}{\rho_{0}+p_{\perp 0}} J_{0}\right] \Gamma .
\end{aligned}
$$

Finally, for the collapse equation used to analyze the instability ranges of a collapsing star in the $\mathrm{N}$ and $\mathrm{pN}$ regimes, we insert all the corresponding values in Eq. (66), and we have

$$
\begin{aligned}
& \frac{\delta T_{0} T_{0}^{\prime}}{\kappa^{2}}-\left(\rho_{0}+p_{r 0}\right)\left[\frac{x r}{c X_{0}}-\frac{X_{0}^{\prime}}{X_{0}}\right]+(\Gamma+1)\left(\frac{X_{0}^{\prime} r}{X_{0} c}\right) \\
& \times\left[\frac{2 c}{r}\left(\rho_{0}+p_{\perp 0}\right)+J_{0}+\frac{c}{r}\left(\rho_{0}+p_{r 0}\right)\right] \\
& +J_{1}-2\left(p_{r 0}-p_{\perp 0}\right)\left(\frac{r}{c}\left(\frac{c}{r}\right)^{\prime}-\frac{1}{r}\right) \\
& +\Gamma\left[\frac{c}{r}\left(p_{r 0}-\frac{\rho_{0}+p_{r 0}}{\rho_{0}+p_{\perp 0}} p_{\perp 0}\right)+\frac{2 c}{r}\left(\frac{\rho_{0}+p_{\perp 0}}{\rho_{0}+p_{r 0}} p_{r 0}-p_{\perp 0}\right)\right. \\
& +\left(\frac{p_{r 0}}{\rho_{0}+p_{r 0}}-\frac{p_{\perp 0}}{\rho_{0}+p_{\perp 0}}\right) \\
& \left.\times J_{0}\right]+\frac{\Gamma r}{c}\left[\frac{c}{r} p_{r 0}+\frac{2 c}{r} \frac{\rho_{0}+p_{\perp 0}}{\rho_{0}+p_{r 0}} p_{r 0}+\frac{p_{r 0}}{\rho_{0}+p_{r 0}} J_{0}\right]_{, 1}=0 .
\end{aligned}
$$

We consider the $\mathrm{N}$ and $\mathrm{pN}$ approximations to obtain the instability ranges in the following.

Newtonian limit

The $\mathrm{N}$ approximations are given by

$X_{0}=1=Y_{0}, \quad \rho_{0} \gg p_{r 0}, \quad \rho_{0} \gg p_{\perp 0}, \quad p_{r 0} \gg p_{\perp 0}$.

Inserting these conditions as well as Eq. (71) in (75), the collapse equation yields

$$
\begin{aligned}
& \frac{\delta T_{0} T_{0}^{\prime}}{\kappa^{2}}-\frac{x r \rho_{0}}{c}+J_{1(\mathrm{~N})}-2 p_{r 0}\left(\frac{r}{c}\left(\frac{c}{r}\right)^{\prime}-\frac{1}{r}\right) \\
& +\Gamma\left[\frac{3 c p_{r 0}}{r}+\frac{3 r}{c}\left(\frac{c p_{r 0}}{r}\right)_{, 1}\right]=0,
\end{aligned}
$$

where

$$
\begin{aligned}
J_{1(\mathrm{~N})}=\frac{r \delta}{c \kappa^{2}} & {\left[\left(e T_{0}-\frac{c T_{0}^{2}}{r}\right)_{, 1}+2\left(\frac{c}{r}\right)^{\prime} T_{0}^{2}-\frac{2 c T_{0}^{2}}{r^{2}}\right.} \\
& \left.+\frac{2 T_{0} e}{r}+\frac{2 c x^{\prime} T_{0}^{\prime 2}}{e r}\right] .
\end{aligned}
$$

To check the instability range for $\mathrm{N}$ approximation, we obtain $\Gamma<\frac{1}{I_{0}}\left[-\frac{\delta T_{0} T_{0}^{\prime}}{\kappa^{2}}+\frac{x r \rho_{0}}{c}-J_{1(\mathrm{~N})}+2 p_{r 0}\left(\frac{r}{c}\left(\frac{c}{r}\right)^{\prime}-\frac{1}{r}\right)\right]$,

where $I_{0}=\frac{3 c p_{r} 0}{r}+\frac{3 r}{c}\left(\frac{c p_{r 0}}{r}\right)_{1}$. This equation expresses the dependence of the adiabatic index on torsion terms along with physical properties such as anisotropic pressure and energy density. As long as the above inequality obtains, the collapsing system stays unstable. For the dynamical instability condition the terms in the collapse equation should be positive in the inequality. It requires that

$-\frac{\delta T_{0} T_{0}^{\prime}}{\kappa^{2}}+\frac{x r \rho_{0}}{c}-J_{1(\mathrm{~N})}+2 p_{r 0}\left(\frac{r}{c}\left(\frac{c}{r}\right)^{\prime}-\frac{1}{r}\right)>0$,

where $I_{0}>0$ holds. In GR [1], the adiabatic index represents a numerical value corresponding to dynamical instability of an isotropic sphere. This is given by the following.

- It is found that the dynamical stability is achieved for $\Gamma>\frac{4}{3}$ when the weight of the outer layers is smaller in relation to the pressure in star.

- The case $\Gamma=\frac{4}{3}$ corresponds to the hydrostatic equilibrium condition.

- When the weight of outer layers increases very fast as compared to the pressure inside the star this gives the collapse and $\Gamma<\frac{4}{3}$ constitutes dynamical instability.

The expressions in Eq. (77) entail the following possibilities:

(i) $\left[-\frac{\delta T_{0} T_{0}^{\prime}}{\kappa^{2}}+\frac{x r \rho_{0}}{c}-J_{1(\mathrm{~N})}+2 p_{r 0}\left(\frac{r}{c}\left(\frac{c}{r}\right)^{\prime}-\frac{1}{r}\right)\right]=I_{0}$,

(ii) $\left[-\frac{\delta T_{0} T_{0}^{\prime}}{\kappa^{2}}+\frac{x r \rho_{0}}{c}-J_{1(\mathrm{~N})}+2 p_{r 0}\left(\frac{r}{c}\left(\frac{c}{r}\right)^{\prime}-\frac{1}{r}\right)\right]<I_{0}$,

(iii) $\left[-\frac{\delta T_{0} T_{0}^{\prime}}{\kappa^{2}}+\frac{x r \rho_{0}}{c}-J_{1(\mathrm{~N})}+2 p_{r 0}\left(\frac{r}{c}\left(\frac{c}{r}\right)^{\prime}-\frac{1}{r}\right)\right]>I_{0}$.

In the first possibility together with Eq. (77), we obtain the instability range as $0<\Gamma<1$. The case (ii) shows that the fraction in Eq. (77) is always less than 1 but depending on the values of the dynamical terms. The case (iii) represents the different numerical values for different values of the dynamical terms. Thus, it shows that the collapsing star 
remains unstable for $\Gamma>1$. Also, Eq. (76) shows that the adiabatic index contains the physical quantities, like $f(R)$ gravity $[6-9,14,15]$.

\section{Post-Newtonian limit}

We consider the $\mathrm{pN}$ approximations in terms of the metric component expressions given by

$X_{0}=1-\frac{m_{0}}{r}, \quad Y_{0}=1+\frac{m_{0}}{r}$,

up to order $\frac{m_{0}}{r}$. Introducing these approximations in Eq. (75), we obtain

$$
\begin{aligned}
& \frac{\delta T_{0} T_{0}^{\prime}}{\kappa^{2}}-\left[\frac{x r}{c}\left(1+\frac{m_{0}}{r}\right)-\frac{m_{0}}{r^{2}}\right]\left(\rho_{0}+p_{r 0}\right)+J_{1(\mathrm{pN})}-2\left(p_{r 0}-p_{\perp 0}\right) \\
& \times\left[\frac{r}{c}\left(\frac{c}{r}\right)^{\prime}-\frac{1}{r}\right]+\frac{(\Gamma+1) m_{0}}{r c}\left[\frac{c}{r}\left(3 \rho_{0}+p_{r 0}+2 p_{\perp 0}\right)+J_{0(\mathrm{pN})}\right] \\
& +\Gamma\left[\frac{c}{r}\left(p_{r 0}-\frac{\rho_{0}+p_{r 0}}{\rho_{0}+p_{\perp 0}} p_{\perp 0}\right)+\frac{2 c}{r}\left(\frac{\rho_{0}+p_{\perp 0}}{\rho_{0}+p_{r 0}} p_{r 0}-p_{\perp 0}\right)\right. \\
& \left.+\left(\frac{p_{r 0}}{\rho_{0}+p_{r 0}}-\frac{p_{\perp 0}}{\rho_{0}+p_{\perp 0}}\right) J_{0(\mathrm{pN})}\right]+\frac{\Gamma r}{c}\left[\frac{c}{r} p_{r 0}+\frac{2 c}{r} \frac{p_{\perp 0}+\rho_{0}}{\rho_{0}+p_{r 0}} p_{r 0}\right. \\
& \left.+J_{0(\mathrm{pN})} \frac{p_{r 0}}{\rho_{0}+p_{r 0}}\right]_{, 1}=0,
\end{aligned}
$$

where $J_{1(\mathrm{pN})}$ and $J_{0(\mathrm{pN})}$ are those terms which belong to the $\mathrm{pN}$ regime in $J_{1}$ and $J_{0}$; the expressions are given by

$$
\begin{aligned}
& J_{0(\mathrm{pN})}=\frac{1}{\kappa^{2}}\left[\delta T_{0} e-\frac{4 c T_{0}^{\prime} \delta}{e r}\left(1-\frac{2 m_{0}}{r}\right)\left\{e^{\prime}-T_{0}^{\prime}\left(x\left(1+\frac{m_{0}}{r}\right)+\frac{2 c}{r}\right)\right\}\right. \\
& +2 \delta\left(1+\frac{2 m_{0}}{r}\right)\left(\frac{c T_{0}^{\prime}}{r}\right)_{, 1}-\frac{4 c \delta T_{0}^{\prime}}{r}\left(1-\frac{m_{0}}{r}\right)\left(\frac{x T_{0}^{\prime}}{e}+\frac{2 m_{0}}{r^{2}}\right) \\
& \left.+\frac{2 c \delta T_{0}^{\prime}}{r}\left(1-\frac{2 m_{0}}{r}\right)\left\{\frac{2}{r}+\frac{m_{0}}{r^{2}}-\frac{2 c T_{0}^{\prime}}{e r}\right\}\right] . \\
& J_{1(\mathrm{pN})}=\frac{r \delta}{c \kappa^{2}}\left[\left(1+\frac{2 m_{0}}{r}\right)\left\{T_{0}\left(e-\frac{T_{0} c}{r}\right)\left(1-\frac{2 m_{0}}{r}\right)\right\}_{, 1}+T_{0}^{2}\left(\frac{c}{r}\right)^{\prime}\right. \\
& +\left(1+\frac{m_{0}}{r}\right)^{\prime}\left(1-\frac{m_{0}}{r}\right)\left(2 T_{0} e-\frac{3 c T_{0}^{2}}{r}+\left(\frac{c}{r}\right)^{\prime} T_{0}^{2}\right)-\frac{2 c T_{0}^{2}}{r^{2}}+\frac{2 T_{0} e}{r} \\
& \left.+\frac{2 c x^{\prime} T_{0}^{\prime 2}}{e r}\left(1-\frac{m_{0}}{r}\right)+\frac{2 c T_{0}^{\prime}}{e r}\left(2 e^{\prime}-x T_{0}^{\prime}\left(1+\frac{m_{0}}{r}\right)-\frac{4 c T_{0}^{\prime}}{r}\right)\right] .
\end{aligned}
$$

Similar to the $\mathrm{N}$ approximation strategy, the instability range is given by

$\Gamma<\frac{\mathcal{A}}{\mathcal{B}}$,

where

$$
\begin{aligned}
\mathcal{A}= & -\frac{\delta T_{0} T_{0}^{\prime}}{\kappa^{2}}+\left[\frac{x r}{c}\left(1+\frac{m_{0}}{r}\right)-\frac{m_{0}}{r^{2}}\right] \\
& \left(\rho_{0}+p_{r 0}\right)-J_{1(\mathrm{pN})}+2\left(p_{r 0}-p_{\perp 0}\right) \\
& \times\left[\frac{r}{c}\left(\frac{c}{r}\right)^{\prime}-\frac{1}{r}\right]-\frac{m_{0}}{r c}\left[\frac{c}{r}\left(3 \rho_{0}+p_{r 0}+2 p_{\perp 0}\right)+J_{0(\mathrm{pN})}\right], \\
\mathcal{B}= & \frac{m_{0}}{r c}\left[\frac{c}{r}\left(3 \rho_{0}+p_{r 0}+2 p_{\perp 0}\right)+J_{0(\mathrm{pN})}\right]+\left[\frac{c}{r}\left(p_{r 0}-\frac{\rho_{0}+p_{r 0}}{\rho_{0}+p_{\perp 0}} p_{\perp 0}\right)\right. \\
& \left.+\frac{2 c}{r}\left(\frac{\rho_{0}+p_{\perp 0}}{\rho_{0}+p_{r 0}} p_{r 0}-p_{\perp 0}\right)+\left(\frac{p_{r 0}}{\rho_{0}+p_{r 0}}-\frac{p_{\perp 0}}{\rho_{0}+p_{\perp 0}}\right) J_{0(\mathrm{pN})}\right] \\
& +\frac{r}{c}\left[\frac{c}{r} p_{r 0}+\frac{2 c}{r} \frac{\rho_{0}+p_{\perp 0}}{\rho_{0}+p_{r 0}} p_{r 0}+\frac{p_{r 0}}{\rho_{0}+p_{r 0}} J_{0(\mathrm{pN})}\right]_{, 1} .
\end{aligned}
$$

We obtain instability ranges of collapsing star with vanishing shear as long as this inequality holds. We see by our analysis that the adiabatic index evinces the dependence of instability ranges on relativistic and torsion terms under a zeroth order configuration.

For the dynamical instability condition, it is required that the right hand side (expressions $\mathcal{A}$ and $\mathcal{B}$ ) in inequality (80) remains positive. Similarly to the $\mathrm{N}$ regime, we have three cases: $\mathcal{A}=\mathcal{B}, \mathcal{A}<\mathcal{B}, \mathcal{A}>\mathcal{B}$. These cases constitute the instability ranges as $0<\Gamma<1$ for the first and second case, while $\Gamma>1$ for the last case.

\section{Concluding remarks}

The collapse in a star occurs due to a state of disequilibrium between inwardly acting gravitational pull and outwardly drawn pressure in the star. In modified theories of gravity, the ranges of dynamical instability depends on the dark source terms in addition to the usual terms (that is, terms of GR) determined by the adiabatic index. We have analyzed the dynamical instability of a collapsing star taking a vanishing shear scalar in ETG gravity. We have taken the interior metric as a general spherically symmetric metric, while the Schwarzschild metric is considered in the exterior region to $\Sigma^{(e)}$ in an anisotropic matter distribution. The contracted Bianchi identities are used to find two dynamical equations corresponding to a star experiencing the collapse process. A well-known power-law ETG model is considered to analyze these ranges. We have used a perturbation strategy on all functions, such as metric components, energy density, pressure components, mass, torsion, and shear scalar, to examine the evolution of collapse with respect to time. We have applied zeroth order and first order perturbed configurations on the field and dynamical equations. The collapse equations have been constructed through the second dynamical equation. The results are given as follows.

The adiabatic index $(\Gamma)$ plays a vital role as we examine the instability ranges for a collapsing star with the shear-free condition. We can analyze the instability regions through this index by applying the $\mathrm{N}$ and $\mathrm{pN}$ approximations on the collapsing equation. In both cases of approximations, we have observed that this index depends upon various quantities, such as the energy density, anisotropy of pressure, and some other constraints. This index shows how we can modify the instability range of a collapsing star under relativistic as well as torsion terms. Hence, the physical properties play a crucial role in analyzing the self-gravitating objects as regards the dynamical instability. Similar to the case of $f(R)$ gravity, the results of the present paper concern physical quantities. It is pointed out that the instability ranges are found as $0<\Gamma<1$ and $\Gamma>1$ for both approximations, i.e., the system shows 
unstable behavior and meets $\Gamma<\frac{4}{3}$ in the $\mathrm{N}$ and $\mathrm{pN}$ perfect fluid limit [1].

We would like to point out here that some authors have discussed the instability analysis by imposing some constraints for finding the solutions [3-9,14-18]. However, we have found the solutions without imposing any extra constraints.

Open Access This article is distributed under the terms of the Creative Commons Attribution 4.0 International License (http://creativecomm ons.org/licenses/by/4.0/), which permits unrestricted use, distribution, and reproduction in any medium, provided you give appropriate credit to the original author(s) and the source, provide a link to the Creative Commons license, and indicate if changes were made. Funded by SCOAP ${ }^{3}$.

\section{References}

1. S. Chandrasekhar, Astrophys. J. 140, 417 (1964)

2. L. Herrera, N.O. Santos, G. Le Denmat, Mon. Not. Roy. Astron. Soc. 237, 257 (1989)

3. H.R. Kausar, J. Cosmol. Astropart. Phys. 01, 007 (2013)

4. R. Kausar, Mon. Not. R. Astron. Soc. 439, 1536 (2014)

5. H.R. Kausar, I. Noureen, Eur. Phys. J. C 74, 2760 (2014)

6. M. Sharif, Z. Yousaf, Mon. Not. R. Astron. Soc. 432, 264 (2013)

7. M. Sharif, Z. Yousaf, Mon. Not. R. Astron. Soc. 434, 2529 (2013)

8. M. Sharif, Z. Yousaf, Eur. Phys. J. C 73, 2633 (2013)

9. M. Sharif, Z. Yousaf, Astropart. Phys. 56, 19 (2014)

10. V.A. Skripkin, Soviet Phys. Doklady 135, 1183 (1960)

11. L. Herrera, G. Le Denmat, N.O. Santos, Gen. Relativ. Gravit. 44, $1143(2012)$
12. M. Sharif, M. Azam, Gen. Relativ. Gravit. 44, 1181 (2012)

13. M. Sharif, M. Azam, Mon. Not. R. Astron. Soc. 430, 3048 (2013)

14. M. Sharif, H.R. Kausar, J. Cosmol. Astropart. Phys. 07, 022 (2011)

15. M. Sharif, Z. Yousaf, Phys. Rev. D 88, 024020 (2013)

16. M. Sharif, R. Manzoor, Astrophys. Space Sci. 354, 2122 (2014)

17. M. Sharif, S. Rani, Mon. Not. Roy. Astron. Soc. 440, 2255 (2014)

18. M. Sharif, S. Rani, Int. J. Theor. Phys. 54, 2524 (2015)

19. E.V. Linder, Phys. Rev. D 81, 127301 (2010). [Erratum: ibid.(82)(2010)109902]

20. P. Wu, H. Yu, Phys. Lett. B 692, 176 (2010)

21. P. Wu, H. Yu, Phys. Lett. B 693, 415 (2010)

22. P. Wu, H. Yu, Eur. Phys. J. C 71, 1552 (2011)

23. M. Sharif, S. Rani, Phys. Rev. D 88, 123501 (2013)

24. M. Sharif, S. Rani, Adv. High Energy Phys. 2014, 691497 (2014)

25. A. Jawad, S. Rani, Eur. Phys. J. C 75, 173 (2015)

26. B. Li, T.P. Sotiriou, J.D. Barrow, Phys. Rev. D 83, 064035 (2011)

27. B. Li, T.P. Sotiriou, J.D. Barrow, Phys. Rev. D 83, 104017 (2011)

28. T.P. Sotiriou, B. Li, J.D. Barrow, Phys. Rev. D 83, 104030 (2011)

29. D. Liu, M.J. Rebouças, Phys. Rev. D 86, 083515 (2012)

30. R. Chan, L. Herrera, N.O. Santos, Mon. Not. R. Astron. Soc. 265, 533 (1993)

31. L. Herrera, N.O. Santos, Astrophys. J. 438, 308 (1995)

32. R. Chan, Mon. Not. Roy. Astron. Soc. 316, 588 (2000)

33. M. Sharif, M. Azam, J. Cosmol. Astropart. Phys. 02, 043 (2012)

34. L. Herrera, D. Di Prisco, J. Ospino, Gen. Relativ. Gravit. 42, 1585 (2010)

35. B.K. Harrison, K.S.Thorne, M. Wakano, J.A. Wheeler, Gravitation Theory and Gravitational Collapse (University of Chicago Press, 1965)

36. R. Chan, K. Kichenassamy, G. Le Denmat, N.O. Santos, Mon. Not. R. Astron. Soc. 239, 91 (1989) 